\title{
DNA synthesis lights up
}

Taking advantage of the fast and highly specific 'click chemistry' reaction, researchers developed a method for detecting DNA synthesis in proliferating cells using small-molecule fluorophores rather than antibodies.

Methods for detecting DNA synthesis are used to assess cell proliferation, cell-cycle kinetics and cell viability. The most common method uses the nucleoside thymidine ana$\log$ 5-bromo-2'-deoxyuridine (BrdU), which incorporates into DNA during $S$ phase of the cell cycle. Cells that were actively replicating their DNA at the time of incubation with BrdU can thus be detected with specific antibodies to BrdU.

Adrian Salic, a cell biologist at Harvard Medical School who confesses to 'dabbling' in chemistry, thought that sending in small molecules to do an antibody's job might have advantages in some situations. "I wanted to be able to look with high-resolution microscopy at DNA in cells," he says. Unfortunately, exposing the BrdU epitope to BrdU antibodies, "involves 'cooking' your tissue with either methanol/acetic acid or concentrated hydrochloric acid," he explains. "Obviously these are harsh conditions for biological specimens, so then the fine microscopic structure looks terrible."

Along with his colleague Timothy Mitchison, Salic decided to harness that well-known bio-orthogonal reaction, the copper(I)-catalyzed [3+2] cycloaddition, or 'click chemistry'. They developed a thymidine analog, 5-ethynyl-2'-deoxyuridine (EdU), containing an alkyne, a functional group not typically found in nature. Proliferating cells incorporate EdU into replicating DNA in place of thymidine. The researchers then incubate the cells with a fluorescent azide molecule, which reacts specifically and rapidly with the alkyne group of EdU to light up the newly synthesized DNA. "Unlike an immunological method, this one is done in minutes," notes Salic. Moreover, it does not require harsh denaturation conditions for fluorescent staining, thus facilitating tissue imaging.

The fluorescent signal resulting from the click chemistry reaction is stronger than that of traditional BrdU immunostaining, which will allow researchers to look at larger sections of tissue with lower magnification. In
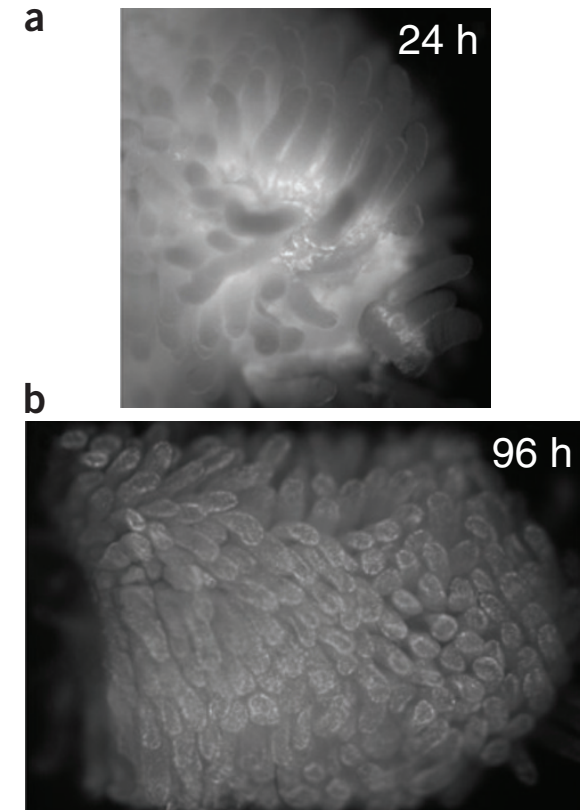

Figure 1 | Cell proliferation in mouse small intestine villi. (a) Detection of EdU labeling after 24 hours, showing high cell proliferation at the base of the villi. (b) Detection of EdU labeling after 96 hours, showing that the labeled cells have moved away from the base to the tips of the villi. Copyright 2008, National Academy of Sciences, USA.

addition, because the fluorescent azide is "500 times smaller than an antibody, it diffuses very easily," explains Salic. "This allows you to look at cell proliferation in a real situation, in a whole tissue or even a whole organ." The researchers showed that proliferating cells from large, fresh sections from mouse small intestine could be imaged to assess cell turnover in the villi (Fig. 1).

Salic also notes that because the assay is fast and requires a minimal number of steps, it should find use in high-throughput applications such as serving as a cell viability assay in drug screening. He is careful to point out, however, that this method will not completely replace the use of BrdU, which has been used successfully for quite some time. The use of EdU will have certainly advantages in many situations, but he says, "it all depends on what sort of experiment you're doing."

\section{Allison Doerr}

\section{RESEARCH PAPERS}

Salic, A. \& Mitchison, T.J. A chemical method for fast and sensitive detection of DNA synthesis in vivo. Proc. Natl. Acad. Sci. USA 105, 2415-2420 (2008). 